\title{
Online Health Information Seeking Behavior of Non-Communicable Disease (NCD) Among Government Employees in Putrajaya Malaysia
}

\author{
AZRIEY MAZLAN \\ NOR AZURA ADZHARUDDIN \\ SITI ZOBIDAH OMAR \\ EZHAR TAMAM \\ Universiti Putra Malaysia
}

\begin{abstract}
Seeking health information is becoming more convenient with various health resources online. It would be beneficial to explore the perception of individuals' health status concerning health information seeking and eHealth literacy. With government employees in Putrajaya found to be at high risk of Non-Communicable Disease (NCD), based on the high rate of obesity, it is beneficial to explore the factors associated with OHISB among this population to ensure adequate attainment and sharing of health information and self-management resources. The Comprehensive Model of Information Seeking (CMIS) is used to measure the factors that influence online health informationseeking behaviour (OHISB). Based on previous studies, the CMIS is commonly used to examine cancer patient's information seeking on health information behaviour. However, this paper will be focusing on government employee's behaviour in using online information as to their health reference. Previous studies have shown that information seeking has resulted in changes in the behaviour of cancer patients. It is believed that the outcome of this study will be beneficial in terms of developing strategies to encourage a healthier lifestyle among this population to prevent the occurrence of NCDs. Therefore, this paper elaborates on some of the theories used in previous studies to enrich individual and community online health information-seeking behaviour and health knowledge dissemination. The findings of this study show the direct relationships between trust in internet health and eHealth Literacy were significant towards OHISB. However, the direct relationships between direct experience, salience, unmet information needs and self-efficacy belief towards OHISB were not significant.
\end{abstract}

Keywords: Comprehensive model of information seeking, online health information seeking behaviour, non-communicable diseases, ehealth literacy, government employees.

\section{INTRODUCTION}

It is a well-established fact that a healthy lifestyle provides tremendous benefits in disease prevention and wellness promotion (World Health Organization, 1999). Unhealthy lifestyles which include an unbalanced diet, sedentary behaviour and smoking are considered major risk factors for non-communicable diseases (Chitson, 1994; Kruger et al., 2005; Steyn \& Damasceno, 2006). To combat these non-communicable diseases, adequate information regarding its risk factors and promotion of healthy lifestyles is essential.

Health information can be sought from various sources which may include interpersonal interactions (e.g family members), electronic media such as the television, printed media and the internet (Galarce, Ramanadhan \& Viswanath, 2011). These additional sources of information, apart from the healthcare providers, may help to satisfy our health information needs (Caiata-Zufferey, Abraham, Sommerhalder \& Schulz, 2010; Czaja, Manfredi, \& Price, 2003; Rozmovits \& Ziebland, 2004; Ziebland et al., 2004). 
Researchers, healthcare organisations and health advocates have used the internet to deliver healthcare services and information efficiently and cost-effectively (Crabb, Rafie, \& Weingardt, 2010; Rideout et al., 2005; Wagner et al., 2004; Wright \& Hill, 2009). A great deal of private and public investment has gone into web-based health interventions and the development of health and medical websites (Crabb et al., 2012).

With the rapid advancements of the internet, health information seeking has become easier for many people. Online resources ranging from information regarding particular diseases to guidance on healthy living are more accessible and readily available. Information can be obtained from various websites, for example, the Ministry of Health website, websites of private healthcare providers or online community forums. Assistance for decision-making seems to be one of the reasons people prefer to retrieve health information independently from the internet. Internet-mediated health resources can enhance individual selfmanagement capabilities and thereby improve important outcomes such as health-related quality of life (Wagner et al., 2004; Jones \& Goldsmith, 2009; Solomon et al., 2012).

\section{RESEARCH BACKGROUND}

In Malaysia, non-communicable diseases such as diabetes, cardiovascular disease and cancer are now accountable for the major causes of mortality and admissions to public hospitals, instead of communicable diseases (Health Facts, 2012). Furthermore, Malaysia ranked first in the prevalence of obesity among South-east Asian countries and sixth among Asian countries. Apart from the complications faced by patients from the diseases, non-communicable diseases also pose a substantial burden in terms of the economy. Therefore, there has been an emphasis on the promotion of healthy lifestyles by the Ministry of Health.

The Ministry of Health Malaysia realises that there is a gap between health knowledge and the health behaviour of the population $(\mathrm{MOH}, 2010)$. In the Strategic Plan for the Ministry of Health Malaysia, one of the pillars is for the usage of information and communications technology (ICT) for health transformation to be further developed. Furthermore, to use internet health information channels to improve public health, there is a need to resolve the divergence between the provision of eHealth and the accessibility of these services to individuals (Norman \& Skinner, 2006).

Based on a national survey, the city in Malaysia with the highest proportion of the overweight and obese population is Putrajaya with $43 \%$ of the population considered obese. Furthermore, according to the survey, government and semi-government employees had the highest percentage of obesity (Institute of Public Health, 2015). Since health information seeking online may help empower people in terms of their health, it would be beneficial to evaluate online health information-seeking behaviour (OHISB) among this population. Currently, it is unknown whether this population has the capability or the confidence to search for, understand, evaluate and apply the health-related information that havhasen obtained from online resources. Without sufficient knowledge, skills, or abilities (Chan \& Kaufman, 2011), government employees may be exposed to non-credible online health information; information that could lead to both intentional and unintentional health risk behaviours (Rice, 2006).

One study in Malaysia involving students in public universities looked at the influence of health literacy on health iinformation-seekingbehaviour among the respondents. It concluded that the influences of health literacy towards health information-seeking behaviour among Malaysian university students were only moderate. This study showed that 
in every 10 university students, 3 of them had a low level of health literacy (Hamzah, Mohamad, \& Abdullah, 2016).

Apart from that, studies regarding internet health information use have primarily focused on public health campaigns and failed to acknowledge how health information flows in mass media or on the internet (Brown \& Walsh-Childers, 2002; Stryker, 2003; Yanovitzky \& Stryker, 2001). Therefore, this paper aims to look into the area of online health informationseeking behaviour that can influence health among the population, particularly among the government employees in Putrajaya, as well as the applicability of the internet as a health information source. Exploring the factors associated with OHISB may provide input for efforts to assist government employees in Putrajaya in obtaining resources such as health information and self-management as well as for them to share the information with others.

Based on the Comprehensive Model of Information Seeking (Johnson, 1997), this study focuses on health information seeking using the online medium which is the internet. It will examine the relationships between demographic characteristics, experiences, salience and belief and OHISB among the respondents. By determining their combined knowledge, confidence and the skill to evaluate and apply electronic health information to health problems, we will be able to predict their OHISB. In turn, this may help in improving the individual's OHISB and subsequently enabling them to obtain relevant health information and thereby encourage healthy lifestyles among them.

This study will answer the question of whether there are significant relationships between OHISB and these five factors: 1) Direct experience of using the internet for health issues; 2) Salient unmet information needs; 3) Self-efficacy in managing health needs; 4) Trust in internet health information, and 5) eHealth literacy. Apart from that, it aims to identify whether there is a mediation effect of internet health information seeking on these hypothesised relationships.

The wide reach of the internet allows health information to be shared with large audiences. Therefore if internet health information can be used as a mediator for better health information seeking among government employees for health decisions, then it will make good sense for the government to promote more online health information to its audience. The results of this study will be able to educate people to increase participation in health information seeking and use the information to make health decisions that will influence their health.

\section{LITERATURE REVIEW}

\section{Internet Health Information Usage}

The internet can be a useful means for people to get health information because individuals can access health information online with convenience and efficiency (Avery et al., 2010; Hayes, 1998). The Internet plays an important role in facilitating the seeking of health information especially by those with physical disabilities or those with lack of mobility (Bundorf et al., 2006). According to the Pew Research Center (Fox \& Duggan, 2013), 72\% of more than 100 million internet users in the U.S. search for health-related information such as disease symptoms, treatment options and medications via the internet.

These individuals use various types of digital devices such as Personal Computers (PCs), Personal Digital Assistants (PDAs) and smartphones to get information with convenience and minimal cost (Mosa, Yoo, \& Sheets, 2012). One of the widely-used models to describe and evaluate information-seeking among the population is the Comprehensive 
Model of Information Seeking (CMIS) which was initially developed for cancer patients (Johnson \& Meischke, 1993).

\section{eHealth}

The revolution of eHealth is evidence of the new era in health information seeking since the introduction of the World Wide Web on the internet. One definition of eHealth relates to its application in changing individuals' lifestyle towards a state of optimal health. Another definition of eHealth is the use of electronically transmitted, stored and retrieved digital data in the health sector for a clinical, educational or administrative purpose (Mitchell, 1999). It also refers to any healthcare delivered electronically over the internet in the form of informational, educational, commercial or direct services by providers or consumers themselves. It allows a more efficient delivery of healthcare (McLendon, 2000).

One of the effective ways to undertake health promotion and health education is with the usage of technology through online health information services or the web-based health information service (WBHIS). WBHIS is a form of online health education with the function of delivering interactive health promotion (Marcus et al., 2000).

The term eHealth does not only refer to the technicalities of the information technology, but also to the way of thinking, attitude and commitment towards a more networked and global approach to address healthcare locally and globally (Eysenbach, 2001). The use of information technology by the public and health care providers to access healthrelated information, support and services also encompass telemedicine and telecare (Wyatt \& Liu, 2002).

Electronic health (eHealth) allows the ease of connection between providers, patients, as well as the government for various purposes such as educating, informing, stimulating innovation and improving the healthcare system (Coach, 2003). Due to the advantages and the potential, it has to offer, the concept of eHealth has grown and gained momentum (Bernhardt et al., 2004). Apart from being a vehicle to improve the transmission of knowledge between health professionals and the public, it also assists patients in improving their health and well-being through closing the communication gap (Kivitis, 2006).

The quality of web-based health information has been examined by several studies concluding that there is a large quality variation between websites and medical information on the web (Kim \& Chang, 2007). The internet plays a vital role in disseminating health information and is becoming an effective medium to facilitate communication to fulfil the public's needs for interaction with health services (McGrath et al., 2007; Hernandez, 2009).

\section{eHealth Literacy}

eHealth literacy comprises six core skills or literacies which are the traditional, media, information, health, science and computer literacies (Norman \& Skinner, 2006). It is based partly on the Social Cognitive Theory and self-efficacy theory (Bandura, 1997). In these theories, competency and confidence are regarded as precursors to behaviour change and skill development (Norman \& Skinner, 2006). eHealth refers to the set of knowledge and skills essential for effective interactions with technology-based health tools (Chan \& Kaufman, 2011). 
Health literacy refers to an individual's knowledge, motivation and competency to access, understand, appraise and apply health information to make decisions on health, disease prevention and health promotion (Sørensen et al., 2012). Computer literacy, on the other hand, refers to the ability to adapt to new technologies effectively and efficiently to solve problems or answer questions through electronic devices (Norman \& Skinner, 2006).

To assess eHealth literacy across the population and contexts, the eHealth Literacy Scale (eHEALS) has been developed. It is a self-report tool and is based on one's perception of his or her skills and knowledge. Researchers may measure the eHealth literacy of a group of people given their familiarity with technology. It may provide a general estimate of the users' eHealth-related skills to be used for health promotion plans or clinical decision-making.

In a Canadian study, 99\% of adolescents had access to the internet with the majority reporting its usage for health in some capacity (Environics Research Group; 2001). Although the quality of this internet access (Skinner, Biscope, \& Poland, 2003) is questionable, this group is more likely to be familiar with and use eHealth than other populations (Skinner, Biscope, Poland, \& Goldberg, 2003). However, despite their familiarity with eHealth, many adolescents are unable to get its full benefit. Many teens have difficulty in engaging with eHealth and understanding health information online despite frequently using it (Gray, Klein, Noyce, Sesselberg, \& Cantrill, 2005). There is a need to navigate the internet with confidence especially concerning health issues where misleading, or false information may result in negative consequences (Eysenbach, 2002).

The tools and resources to evaluate health information online and critically appraise eHealth resources may provide the opportunity to empower and protect the consumers (Eysenbach, \& Jadad, 2001). The eHEALS has shown good prospects in measuring eHealth literacy. It has a high internal consistency and modest test-retest reliability, suggesting its utility in assessing eHealth literacy over time and evaluating intervention outcomes. It is easily administered and thus can be used independently or incorporated with other measures of health.

\section{Healthy Lifestyles}

One important determinant of the health status of an individual is a lifestyle. According to the World Health Organization (WHO), up to $60 \%$ of a person's health-related quality of life depends on his/her lifestyle (Ziglio, Currie, \& Rasmussen, 2004). Numerous publications have shown that healthy lifestyles can reduce mortality and morbidity (Krueger and Chang, 2008; Nothlings, Ford, Kroger, \& Boeing, 2010).

The promotion of a healthy lifestyle and wellness has been the main focus of the Ministry of Health Malaysia ( $\mathrm{MOH}$ ). Healthy lifestyles depend on the early adoption of healthy living habits with unhealthy lifestyles among adolescents being found to be carried on to adulthood (Lowry, Galuska, Fulton, Wechsler, Kann, \& Collins, 2000). Urbanisation, globalisation and changes in dietary practices have led to unhealthy lifestyle and habits ( Popkin and Gordon- Larsen, 2004; Candib, 2007). Unhealthy behaviours due to urbanisation and globalisation changes put the population at risk of chronic non-communicable diseases. Behaviours related to health in the early stages of life have an impact on the development of diseases, especially non-communicable disease, later in life. Many risk factors for these diseases are modifiable and can be addressed at an early stage (Gall, Jamrozik, Blizzard, Dwyer, \& Venn, 2009). 
It is therefore essential to improve healthy behaviours and lifestyles among the population. Studies among university students have shown that the majority of them are engaged in health risk behaviours such as smoking, alcohol abuse, substance abuse and physical inactivity (Irwin, 2007; Lee \& Loke, 2005).

\section{Online Health Information Seeking Behaviour (OHISB)}

The majority of adults seek health information to improve their quality of life (Sharit, Hernandez, Nair, Kuhn, \& Czaja, 2011). An individual can be considered to have OHISB if they retrieve health information from the internet. Factors contributing to this behaviour include the type of information sought, reasons for seeking the information and the individual's level of experience (Lorence, Park \& Fox, 2006).

Based on previous studies, health information seeking can be conceptualised and measured through the different dimensions of amount and frequency (Johnson \& Meischke, 1993) for example time spent on searching information sources (Shaw et al., 2008), the number of health topics sought and the number of information sources used (Nagler et al., 2010).

Previous studies on health information seeking focus on OHISB only as an outcome and examine the effects of predictors of OHISB. For example, demographic characteristics of age, gender and education significantly predict OHISB. Among various demographic characteristics, operationalisation of age groups (e.g. adolescences and the elderly) has varied across studies. For example, studies of the influence of older age with OHISB have variously examined individuals at or above age 60 (Bass et al., 2006) and 65 (Lee et al., 2008). In such diversity, it is difficult to conclude age from the existing literature. Previous studies have also revealed that direct experiences related to health conditions such as cancer and self-rated health significantly predict OHISB. However, research findings were mixed and at times conflicting.

Some studies showed a significant relationship between years since cancer diagnosis and OHISB (Basch et al., 2004; Chou et al., 2011), while others did not show a significant relationship (Fogel et al., 2002; Helft et al., 2005). In terms of self-rated health, various studies have also reported mixed results. Some researchers revealed a significant relationship between better self-rated health and higher OHISB (Walsh et al., 2010). However, other researchers reported results in the opposite direction where worse self-rated health predicted higher OHISB (Atkinson, Saperstein, \& Pleis, 2009; Baker et al., 2003; Chou et al., 2011; Oh \& Cho, 2015; Xiao et al., 2014).

Based on previous literature on cancer survivors as well as elderly populations, the relationship between trust in internet health information and OHISB has been examined relatively less often in previous literature compared to the relationship between trust in other information sources of trust in health professionals and OHISB.

\section{Comprehensive Model of Information Seeking (CMIS)}

Johnson's (1997) Comprehensive Model of Information Seeking (CMIS) has been widely used in literature for health information and its seeking (Case, Andrews, Johnson, \& Allard, 2005; Rice, McCreadie, \& Chang, 2001). In the context of seeking information related especially to cancer, CMIS has six factors; demographics, direct experience, self-efficacy, salience, information carrier characteristics and online health information seeking (Johnson \& Meischke, 1993). 
The model has originally been applied in researches related to health communication, particularly cancer-related information seeking. Nonetheless, it has been widely applied in the setting of organisational communication as well (Johnson, Donohue, Atkin, \& Johnson, 1995). In communication at the organisational level, the antecedents have direct effects (Johnson, 1997). The CMIS can also examine health information seeking (HIS) via the internet.

The internet contains various types and forms of health information with varying levels of credibility (Cline \& Haynes, 2001) and individuals with particular health issues or concerns may decide based on factors such as relationship demands, cultural situations and other contextual concerns (Brashers et al., 2002).

One of the strengths of Johnson's CMIS is that it is a very general model which can be applied in different contexts of information seeking. The factors contained in the model are useful in assessing the behaviour of health information seeking for online health information among a particular population. It has been widely used in studies involving patients especially cancer patients.

Among internet users with chronic illnesses or disabilities, more than $87 \%$ have used the internet to look for health information online (Fox \& Fallows, 2003). In a study involving more than 10,000 patients with chronic illnesses, women spent more time looking for health information online compared to men (Millard \& Fintak, 2002).

\section{a. Demographic Characteristics}

Studies also have found that demographics such as age, gender and ethnicity do contribute to some extent to health information-seeking behaviour, which is consistent with the CMIS. A previous study also found that gender was a significant predictor of online information seeking whereby male cancer patients engaged in greater OHISB than females (Robinson et al., 2006).

\section{b. Direct Experience}

This generally refers to an individual's degree of personal experience with a particular disease themselves or through someone in their network (e.g. time since cancer diagnosis) or their general health conditions (e.g. self-rated health) (Johnson et al., 2001; Johnson \& Meischke, 1993). Experience can be measured through the individuals' perceived symptoms related to the disease, their experienced pain and their concerns about the disease (Johnson \& Meischke, 1993).

\section{c. Salience of Information}

Salience means personal significance and interests (Johnson \& Meischke, 1993). According to the CMIS, individuals are usually motivated to search for information when they realise that they are missing some salient information (Case et al., 2005) and an insufficient information environment has been regarded as a factor for salience. Previous studies have measured salience through the individuals' perceived needs and a necessity for information and assistance of others (Johnson et al., 1995). The concept of salience supports the view that information seeking is greatly influenced by an individual's unfulfilled needs for health information. 


\section{d. Beliefs (Self Efficacy)}

An individual's health beliefs may strongly affect information carrier factors such as trust in information sources and the individuals' exposure to information sources. According to the CMIS, beliefs refer to an individual's feeling of self-efficacy, which is "the extents to which individuals perceive they have control over the future or perceive that there are efficacious methods of prevention, treatment, and control" (Johnson et al., 2001). The models suggest that if individuals have a low sense of self-efficacy or believe that knowing more about the disease will not allow them to solve their problems, they are less likely to seek health information. Perceptions of powerlessness can lead to insufficient health-related knowledge (Caiata-Zufferey et al., 2010; Leydon et al., 2000) because people have no motivation to learn more about their health condition when they feel like they have no control over it (CaiataZufferey et al., 2010).

\section{e. Information Carrier Characteristics}

Information carrier characteristics can influence health information-seeking behaviour directly as well as indirectly (through utility) (Johnson \& Meischke, 1993). A study involving traditional media by Johnson and Meischke (1993) found that based on the CMIS, the strongest relationship is between information carrier characteristics and utility. The role of information carrier characteristics has also been found to be significant in the context of online media.

\section{METHODOLOGY}

This research is a quantitative survey-based study and is guided by clearly stated research problems and hypotheses. Utilising a self-administered questionnaire, it is aimed to investigate the relationship between OHISB and its associated factors (salience, direct experience, beliefs, trust in the internet, eHealth literacy) as well as the mediating effect of the internet health information seeking.

\section{Study Sample}

The respondents consist of 379 government employees from five government ministries in Putrajaya. One ministry was selected from each of the five parcels in Putrajaya (parcel 1,2,3,4 and 5). The sample size was calculated based on the formula by Krejcie, Robert, Morgan, and Daryle (1970) for continuous and categorical variables.

Table 1: Total Population of Government Employee Representing Putrajaya From five Ministries

\begin{tabular}{lcc}
\hline Ministry & $\begin{array}{c}\text { Number Of } \\
\text { Government } \\
\text { Employees }\end{array}$ & $\begin{array}{c}\text { Total Taken For } \\
\text { Research }\end{array}$ \\
\hline ICU & 250 & 13 \\
PMD & 700 & 37 \\
PSD & 1300 & 67 \\
MOHA & 1350 & 70 \\
MOH & 3700 & 192 \\
Total & $\mathbf{7 3 0 0}$ & $\mathbf{3 7 9}$ \\
\hline
\end{tabular}


Pilot Test

A pilot study was conducted in the Ministry of Health Malaysia involving 30 respondents where it took them 20-30 minutes to complete the questionnaire. Analysis based on the pilot test yielded a Cronbach Alpha of 0.773-0.944 (Refer Table 2). Reliability coefficient or Cronbach Alpha is commonly used to test the internal consistency of the instrument of the study (Norusis, 1990). The coefficient value of the measurement scale is sensitive to the numbers of items in the scale, sample size and a value of at least 0.7 is considered reliable (Nunnally, 1978; Pallant, 2001; Hair et al., 2010).

Table 2: Measurement Reliability Analysis Pre-Test Results $(n=30)$

\begin{tabular}{lcc}
\hline \multicolumn{1}{c}{ Variable } & Item Number & Cronbach's Alpha \\
\hline Information Carrier Characteristics & $\mathrm{Q} 9.1-\mathrm{Q} 9.5$ & 0.944 \\
Internet Health Information Seeking & $\mathrm{Q} 10.1-\mathrm{Q} 10.11$ & 0.888 \\
Direct Experience Using Internet & $\mathrm{Q} 11.1-\mathrm{Q} 11.5$ & 0.891 \\
Salience & $\mathrm{Q} 12.1-\mathrm{Q} 12.4$ & 0.880 \\
Internet Self-efficacy & & \\
(Belief) & $\mathrm{Q} 13.1-\mathrm{Q} 13.8$ & 0.931 \\
eHealth Literacy & $\mathrm{Q} 14.1-\mathrm{Q} 14.8$ & 0.952 \\
Online Health Information Seeking & & \\
Behavior (OHISB) & $\mathrm{Q} 15.1-\mathrm{Q} 15.5$ & 0.772 \\
\hline
\end{tabular}

\section{Actual Result}

This study had used Structural Equation Modeling (SEM) to test the hypothesised relationships between the predictors and OHISB. The structural model of this study is supported by Confirmatory Factor Analysis (CFA). Based on the research, all the variables in this study were items that are taken from previous studies and were adapted to the research framework.

After running all the tests, the result showed that the direct relationships between trust in internet health information (ICC) and OHISB were significant. The direct relationships between internet health information seeking (ICUF) and OHISB were significant and belief in internet self-efficacy and OHISB was also significant. However, the direct relationships between direct experience and salience of unmet information on OHISB were not significant. These results are displayed in Table 3.

Table 3: Results of SEM on Effect of Predictor on OHISB.

\begin{tabular}{lccccc}
\hline \multicolumn{1}{c}{ Construct } & $\boldsymbol{\beta}$ & Beta & S.E & CR & $\boldsymbol{p}$ \\
\hline OHISB<---Direct Experience & 0.021 & 0.023 & 0.066 & 0.351 & 0.726 \\
OHISB <--- Salience & 0.026 & 0.021 & 0.045 & 0.475 & 0.635 \\
OHISB <--- Belief & -0.125 & -0.108 & 0.048 & -2.239 & 0.025 \\
OHISB <--- ICC & 0.206 & 0.212 & 0.062 & 3.431 & 0.000 \\
OHISB <--- ICUF & 0.272 & 0.253 & 0.062 & 4.099 & 0.000 \\
\hline
\end{tabular}

Based on the measurement model (Figure 1), all five indices indicate a good fit (Table 4). It can be inferred that the observed variables (items) can identify the unobserved variables (constructs) and thus can measure the theoretical model. Based on the analysis, the structural model met the model fit indices. 
Online Health Information Seeking Behavior of Non-Communicable Disease (NCD) Among Government Employees in Putrajaya Malaysia

Azriey Mazlan, Nor Azura Adzharuddin, Siti Zobidah Omar \& Ezhar Tamam

Table 4: Fit Indices for Structural Model

\begin{tabular}{lccc}
\hline Fit Index & Threshold & Value & Conclusion \\
\hline X2 Chi-sq & P-value $\leq 5.0$ & 1.844 & Fit \\
GFI & $\geq 0.9$ & 0.885 & Fit \\
NFI & $\geq 0.9$ & 0.907 & Fit \\
CFI & $\geq 0.9$ & 0.955 & Fit \\
IFI & $\geq 0.9$ & 0.955 & Fit \\
RMSEA & $\leq 0.08$ & 0.047 & Fit \\
\hline
\end{tabular}

Х2: relative chi-square); GFI: goodness of fit; NFI: Normal Fit Index; IFI: Incremental fit index, CFI: Comparative Fit Index; RMSEA: Root Mean Squared Error of Approximation

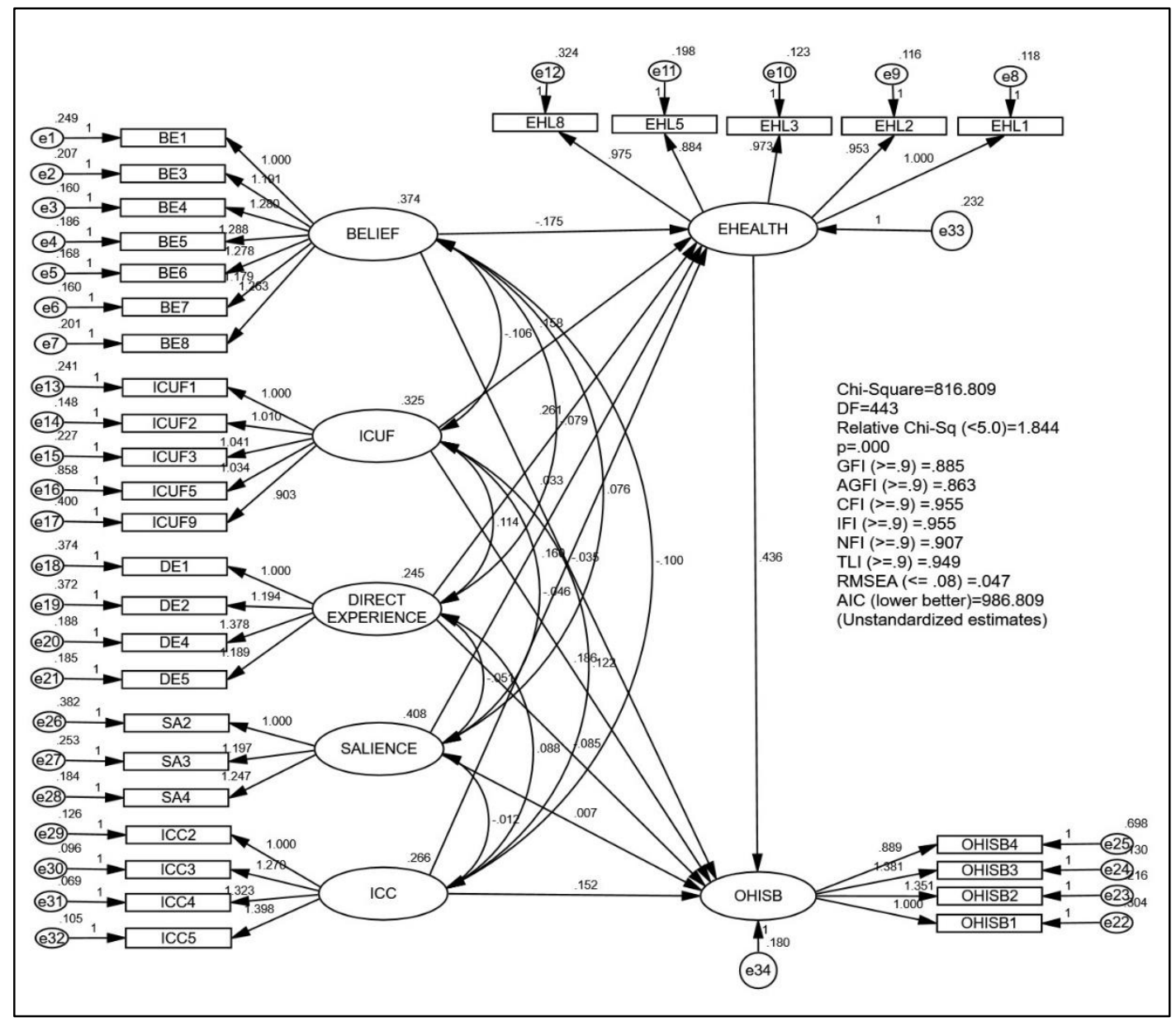

Figure 1: The Model of the Study

\section{RESULTS AND DISCUSSION}

The present study was carried out to explore the effect of information-seeking behaviour on health information seeking using the internet which was based on current literature and theory.

This study is important as we can determine the co-existence between human communication and technology used for health information seeking. The internet, which acts as a useful tool to retrieve information, makes it the best source to seek health information. The abundance of free health information on the internet can make health information seeking easier for the health information seeker. A study by Joorabchi et al. (2013) stated that usage of the internet has improved remarkably and has turned out to be an important part of people's daily lives. 
A previous study by Pew Research Center stated that people having no chronic disease are less interested in searching for health information through the internet compared to those with chronic diseases (Fox \& Duggan, 2013). This shows that there are different patterns of search behaviour between those with specific health conditions and those seeking general online health information.

This topic produces a significant understanding on the reliability of internet usage and eHealth services in providing vast information related to health from an individual and community perspective. This is essential to establish connections between people in a society, especially government employees, and to justify the effects of health information-seeking behaviour communication. Online health information seeking among government employees is important for their health and also to portray a good example of healthier lifestyles among government employees and the community.

Even so, this mechanism will not work if individuals or communities do not want to change or improve their behaviour in online information search especially in regards to health. The connection between devices and human behaviour is needed to produce the desired impact on health behaviour. A study by Zhao (2009) stated that people who can browse health information via the internet conveniently and at a higher speed would increase their OHISB, especially for home internet users. The indication of this result shows that people who receive good internet services are more likely to improve in their OHISB.

It also essential to consider the other factors associated with the level of eHealth literacy knowledge and usage of the internet when searching for health information. This present study focused only on the individual's internet usage and level of eHealth literacy in searching for health information without exploring other health communication experiences that can benefit other users of the system. In a previous study by Van Deursen and Van Dijk (2011), it was found that the eHEALS instrument does not fully demonstrate the eHealth literacy competencies. Instead, it projects an individual's perception of personal knowledge and skills related to eHealth literacy. This shows that it is still unclear how accurately government employees measure and evaluate health information that they retrieve from the internet. Another possible benefit of internet health information seeking is to blur the boundaries between the person and the online health information provider.

Online health information seeking offers a good opportunity to engage and test for eHealth literacy because of its unique six-component facets that constitute the set of core skills and knowledge. Internet worldwide statistics (2016) stated that over 3.6 billion people use the internet globally. High-speed internet access and its affordability to subscribe for the service have increased the percentage of people using the internet for health-related information searching.

Apart from using the internet as a personal source of health information seeking, the internet can also be used as a medium to gather health information by sharing and exchange experiences with other users by communicating directly with doctors or health practitioners and interacting with other people about experiences on health-related issues. Hence, participating in good health information seeking and being eHealth literate is not only for selfbenefit, it is also for sharing knowledge and experiences that can benefit a wider circle of people - friends, family and community.

In view of these findings, there is a need for the government, especially the Ministry of Health Malaysia to seriously initiate intervention programs in the workplace to identify and manage government employees health conditions. Although many programs and 
government funds are spent in acknowledging the importance of healthy lifestyles through websites or health portals, the effectiveness of these programs has yet to be studied. Whether or not these programs deliver the target impact towards government employees still need to be examined.

Furthermore, individuals must be willing to transform and equip themselves with internet self-efficacy skills as it is an important step toward enhancing their OHISB. Higher OHISB will lead them to a higher level of information seeking and gain better prospects to manage their health conditions.

\section{CONCLUSION}

Based on this research, it can be concluded that the internet and eHealth Literacy is important in all forms of online health information seeking, regardless of the sources or medium involved. eHealth literacy skills are essential in ensuring the health information retrieved from specific health portals or websites are relevant to the interest of the audience and serve the purpose of online health information seeking. The diversity of free health information available on the internet can make health information seeking easier for the health information seeker. This shows that the longer and more frequently people use the internet, the more likely their online health information-seeking behaviour level will improve and their exploration of information will be more selective and composed.

There are some practical implications in terms of interventions in health information seeking towards building healthy lifestyles. To enhance the search for online health information, the government could invest in initiatives to motivate and encourage government employees to use the internet more frequently. In other words, government employees should be encouraged to use the internet not only for health information seeking but also for other forms of information search. To ensure the success of these initiatives, the government with the supervision of the Ministry of Health should look closely into their current mechanisms (e.g., MyHealth Portal, MyHealth Mobile) to attract more participation not only from government employees but the rest of the workforce in Malaysia. This is important to create a sense of responsibility to be health literate not only for self-related purposes but for sharing with other people.

BIODATA

Azriey Mazlan is a final year student under PhD Programme at University Putra Malaysia. Email: azrieymazlan@yahoo.com

Nor Azura Adzharuddin is a senior lecturer at University Putra Malaysia. Email: zurh@upm.edu.my

Siti Zobidah Omar is a senior lecturer at University Putra Malaysia. Email: zobidah@upm.edu.my

Ezhar Tamam is a senior lecturer at University Putra Malaysia. Email: ezhar@upm.edu.my 


\section{REFERENCES}

Avery, E., Lariscy, R., Amador, E., Ickowitz, T., Primm, C., \& Taylor, A. (2010). Diffusion of social media among public relations practitioners in health departments across various community population sizes. Journal of Public Relations Research, 22(3), 336-358.

Brown, J., \& Walsh-Childers, K. (2002). Effects of media on personal and public health. In J. Bryant, \& D. Zillman (Eds.), Media effects: Advances in theory and research (pp. 453488). Lawrence Erlbaum Associates.

Bundorf, M. K., Wagner, T. H., Singer, S. J., \& Baker, L. C. (2006). Who searches the internet for health information? Health Services Research, 41(3p1), 819-836.

Caiata-Zufferey, M., Abraham, A., Sommerhalder, K., \& Schulz, P. J. (2010). Online health information seeking in the context of the medical consultation in Switzerland. Qualitative Health Research, 20(8), 1050-1061.

Case, D. O., Andrews, J. E., Johnson, J. D., \& Allard, S. L. (2005). Avoiding versus seeking: the relationship of information seeking avoidance, blunting, coping, dissonance, and related concepts. Journal of the Medical Library Association, 93(3), 353-362.

Chan, C. V., \& Kaufman, D. R. (2011). A framework for characterizing eHealth literacy demands and barriers. Journal of Medical Internet Research, 13(94).

Chitson, P. (1994). Health care implications of non-communicable diseases. Proceedings of the IGU Conference on Health Problems.

Crabb, J., Stewart, R. C., Kokota, D., Masson, N., Chabuya, S., \& Krishnadas, R. (2012). Attitudes towards mental illness in Malawi: A cross-sectional survey. BMC Public Health 2012, 12(541).

Fox, S., \& Duggan, M. (2013). Information triage. Pew Research Center. Pew Internet and American Life Project. https://www.pewresearch.org/internet/2013/01/15/

Galarce, E. M., Ramanadhan, S., Viswanath, K. (2011). Health information seeking. In T. L. Thompson, R. Parrot, \& J. F. Nussbaum (Eds.), The Routledge handbook of health communication 2nd edition (pp. 161-180). Routledge.

Hamzah, M. R., Mohamad, E., \& Abdullah, M. Y. (2016). Pengaruh literasi kesihatan terhadap tingkah laku pencarian maklumat kesihatan dalam kalangan pelajar universiti awam. Jurnal Komunikasi: Malaysian Journal of Communication, 32(2), 405-424.

Hartoonian, N., Ormseth, S. R., Hanson, E. R., Bantum, E. O., \& Owen, J. E. (2014). Informationseeking in cancer survivors: Application of the comprehensive model of informationseeking to HINTS 2007 data. Journal of Health Communication, 19(11), $1308-1325$.

Hayes, M. G. (1998). Individuals with disabilities using the internet: A tool for information and communication. Technology and Disability, 8(3), 153-158.

Institute for Public Health (IPH). (2015). National health and morbidity survey (NHMS) 2015. National Institutes of Health, Ministry of Health Malaysia.

Jones, R., \& Goldsmith, L. (2009). What is the evidence for the benefits and outcomes for digital health services? [Final Report to NHS Choices Plymouth, University of Plymouth, England].

Johnson, J. D., Donohue, W. A., Atkin, C. K., \& Johnson, S. (1995). A comprehensive model of information seeking. Science Communication, 16(3), 274-303.

Johnson, J. D., \& Meischke, H. (1993). A comprehensive model of cancer-related information seeking applied to magazines. Human Communication Research, 19(3), 343-367. 
Johnson, J. D., Andrews, J. E., \& Allard, S. (2001). A model for understanding and affecting cancer genetics information seeking. Library \& Information Science Research, 23(4), 335-349.

Krejcie, R. V., \& Morgan, D. W. (1970). Determining sample size for research activities. Educational and Psychological Measurement, 30, 607-610.

Kruger, S. H., Puoane, T., Seneka, M., \& Van der Merwe, T. M. (2005). Obesity in South Africa: Challenge for government and health professionals. Public Health Nutrition, 8(5), 491500.

Kutner, M., Greenberg, E., Jin, Y., \& Paulsen, C. (2006). The health literacy of America's adults: Results from the 2003 national assessment of adult literacy (NCES 2006-483). U.S. Department of Education. National Center for Education Statistics.

Koh, H. K., \& Rudd, R. E. (2015). The arc of health literacy. Journal of American Medical Association, 314(12), 1225-1226.

Lorence, D. P., Park, H., \& Fox, S. (2006). Assessing health consumerism on the web: A demographic profile of information-seeking behaviours. Journal of Medical Systems, 30(4), 251-258.

Ministry of Health Malaysia. (2012). Health facts. Health Informatics Centre, Planning and Development Division.

Ministry of Health Malaysia. (2010). National strategic plan for non-communicable disease (NSPNCD): Medium term strategic plan to further strengthen the cardiovascular diseases and diabetes prevention and control program in Malaysia (2010-2014). Disease Control Division, Ministry of Health Malaysia.

Mosa, A. S. M., Yoo, I., \& Sheets, L. (2012). A systematic review of healthcare applications for smartphones. BMC Medical Informatics and Decision Making, 12(67).

Nagler, R. H. (2010). Differences in information seeking among breast, prostate, and colorectal cancer patients: Results from a population-based survey. Patient Education and Counselling, 81(1), S54-S62.

Neter, E., \& Brainin, E. (2012). eHealth literacy: Extending the digital divide to the realm of health information. Journal of Medical Internet Research, 14(1), e19.

Norman, C. D., \& Skinner, H. A. (2006). eHealth Literacy: Essential skills for consumer health in a networked world. Journal of Medical Internet Research, 8(2), e9.

Rice, R. E. (2006). Influences, usage, and outcomes of Internet health information searching: Multivariate results from the Pew surveys. International Journal of Medical Informatics, 75(1), 8-28.

Rice, R. E., McCreadie, M., \& Chang, S. L. (2001). Accessing and browsing information and communication. MIT Press.

Ruppel, E. K. (2016). Scanning health information sources: Applying and extending the comprehensive model of information seeking. Journal of Health Communication, 21(2), 208-216.

Robinson, J., Thompson, T., \& Tian, Y. (2006). Seeking cancer information on the internet: Antecedents and satisfactions (conference paper). Annual conference of the International Communication Association, Dresden, Germany.

Sharit, J., Hernandez, M. A., Nair, S. N., Kuhn, T., \& Czaja, S. J. (2011). Health problem solving by older persons using a complex government web site: Analysis and implications for web design. ACM Transactions on Accessible Computing (TACCESS), 3(3). 
Shaw, B. R., Dubenske, L. L., Han, J. Y., Cofta-Woerpel, L., Bush, N., Gustafson, D. H., \& McTavish, F. (2008). Antecedent characteristics of online cancer information seeking among rural breast cancer patients: An application of the cognitive-social health information processing (C-SHIP) Model. Journal of Health Communication, 13(4), 389408.

Solomon, M., Wagner, S.L., \& Goes, J. (2012). Effects of a web-based intervention for adults with chronic conditions on patient activation: Online randomized controlled trial. Journal of Medical Internet Research, 14(1), e32.

Stryker, J. E. (2003). Media and marijuana: A longitudinal analysis of news media effects on adolescents' marijuana use and related outcomes, 1977-1999. Journal of Health Communication, 8(4), 305-328.

Steyn, K., \& Damasceno, A. (2006). Lifestyle and related risk factors for chronic diseases. In D. T. Jamison, R. G. Feachem, W. M. Makogoba, R. E. Bos, K. F. Baingana, J. K. Hofman, \& K. O. Rogo (Eds.), Disease and mortality in sub-saharan Africa (pp. 247-264). The World Bank.

Torpy, J. M., Burke, A. E., \& Golub, R. M. (2011). Health Literacy. Journal of American Medical Association, 306(10), 1158.

Wagner, T. H., Baker, L. C., Bundorf, K., \& Singer, S. (2004). Use of the internet for health information by the chronically ill. Preventing Chronic Disease, 1(4).

World Health Organization. (1999) Healthlliving: What is a healthy lifestyle?. Copenhagen: WHO Regional Office for Europe. http://www.who.int/iris/handle/10665/108180

Yanovitzky, I., \& Stryker, J. (2001). Mass media, social norms, and health promotion effects. Communication Research, 28(2), 208-239. 